\title{
Cirugía de las hernias: efectividad y coste para el sostenimiento del sistema sanitario
}

\author{
Julio Miguel Vila y Blanco ${ }^{1,2}$ y Eva Jiménez González de Buitrago ${ }^{3}$
}

\section{Hernia surgery: effectiveness and cost for the maintenance of the health system}

Introduction: Hernias are very frequent pathologies in a General Surgery Service (GSS); its treatment and costs are very different, depending on the surgeon, if there is admission ( $\mathrm{SH}$ ) or major outpatient surgery (MOS). A) Main objective. Study costs of (non-pericolostomic) abdominal wall hernia surgical procedures in the GSC (cost-outcome ratio and the cost-efectiveness). Materials and Method: Descriptive, longitudinal, observational and retrospective study. Abdominal wall hernias treated between 1st October, 2015 and 30th September, 2016; after surgery follow up until 30th October, 2016 (1 to 12 months). In total 370 hernia surgeries were performed. Results: $79.4 \%$ of males, mean 59.95 years, $51.90 \%$ of ASA II, 55.8\% local anesthesia, 59.72\% inguinal hernias, 36.94\% indirect unilateral inguinal hernias, 55.17 minutes for surgeon, $54.44 \%$ for MOS, 4.77 days of average stay in admitted patients. Perioperative complications $2.3 \%$, early $4.8 \%$ (with 5 reoperations) and late $12.8 \%$ ( 3 reinterventions due to relapse). $95.41 \%$ discharge, mean time 6.59 weeks. Material costs vary from $€ 109.87$ (simple inguinal hernia) to $€ 370.41$ (eventrations). Median surgery room cost $€ 338.80$. Cost/day MOS $€ 807.30$ and with income $€ 1056.03$. Median cost of simple inguinal hernia $€ 422.69$ and eventration $€ 709.89$. Median cost due to inguinal hernia complication $€ 1405.81$ and eventration $€ 8350.88$. Median cost per process $\operatorname{MOS} € 1213.98$ and that of $\mathrm{SH} € 3689.80$. Conclusion: The interventions of simple unilateral inguinal hernia, crural and umbilical, using a tension-free technique, prosthetic material, without drainage, MOS, local anesthesia (with/without sedation) and without complications are the most cost-effective (better cost-benefit and cost-effectiveness ratio).

Key words: abdominal wall hernias; cost-result; cost-effectiveness.

\section{Resumen}

Introducción: Las hernias son patologías muy frecuentes en un Servicio de Cirugía General (SCG); su tratamiento y costes son muy diferentes, dependiendo del cirujano, si hay hospitalización $(\mathrm{CH})$ o cirugía mayor ambulatoria (CMA). Objetivo principal es el estudio de costes-resultados y de coste-efectividad de las hernias de pared abdominal (no pericolostómicas) realizadas por el SCG. Materiales y Método: Estudio descriptivo, longitudinal, observacional y retrospectivo. Intervenidas 370 hernias de pared abdominal, del 1 de octubre de 2015 al 30 de septiembre de 2016; seguimiento postquirúrgico hasta el 30 de octubre 2016 (1 a 12 meses). Resultados: 79,4\% varones, media 59,95 años, 51,90\% ASA II, 55,8\% anestesia local, $59,72 \%$ hernias inguinales, $36,94 \%$ hernias inguinales unilaterales indirectas, 55,17 minutos por intervención, 54,44\% por CMA, 4,77 días de estancia media en ingresados. Complicaciones perioperatorias $2,3 \%$, tempranas $4,8 \%$ ( 5 reintervenciones) y tardías $12,8 \%$ ( 3 reintervenciones por recidiva). Altas 95,41\%, tiempo medio 6,59 semanas. Coste de material de 109,87 € (hernia inguinal simple) hasta 370,41 $€$ (eventración). Coste mediana quirófano 338,80 €. Coste/día CMA 807,30 $€$ y con ingreso 1056,03 $€$. Mediana coste de hernia inguinal simple 422,69€ y de eventración 709,89€. Mediana coste por complicación de hernia inguinal $1405,81 €$ y de eventración $8350,88 €$. Mediana coste por proceso con CMA $1213,98 €$ y con ingreso 3689,80 €. Conclusión: Intervenciones de hernia inguinal unilateral simple, crural y umbilical, con técnica libre de tensión, material protésico, sin drenaje, CMA, anestesia local (con/sin sedación) y sin complicaciones resultan las más coste-efectivas (mejor relación coste-beneficio y coste-efectividad). Palabras clave: hernias pared abdominal; coste-resultado; coste-efectividad.

\author{
'Cirugía General y Aparato \\ Digestivo Hospital \\ Universitario Virgen de la Luz \\ Cuenca. España. \\ ${ }^{2}$ Cirugía General y Aparato \\ Digestivo Hospital \\ Universitario Infanta Leonor. \\ Madrid, España. \\ ${ }^{3}$ Medicina Preventiva Hospital \\ Universitario Infanta Leonor. \\ Madrid, España. \\ Recibido el 10 de marzo \\ de 2019 y aceptado para \\ publicación el 20 de agosto \\ de 2019.
}

Correspondencia a: Dr. Julio Miguel Vila y Blanco juliomiguel.vila@salud. madrid.org 


\section{Introducción}

Uno de los objetivos más importantes de las políticas de sanidad es proveer servicios médicos a los ciudadanos. Y siendo los recursos insuficientes, para satisfacer todas sus necesidades en salud, deben alcanzarse los mejores resultados en calidad, eficiencia, adecuación de la actividad asistencial y uso de recursos sanitarios. Y ello es aplicable a la patología herniaria de la pared abdominal, que induce un consumo de recursos humanos, materiales y económicos muy importante; aunque su prevalencia e incidencia en la población general es variable, es una de las intervenciones más realizadas en un Servicio de Cirugía General (SCG) ${ }^{1-5}$.

Por ello, el nexo entre las necesidades de atención sanitaria y las realidades presupuestarias (puestas de manifiesta en la época de crisis pasada, tras una mala planificación y gestión sanitaria de años anteriores), ha propiciado el control del gasto, la reducción de costes de ineficiencias (mayor eficiencia) y mejoras en la productividad.

Por ello, hay que comprobar los beneficios económicos del tratamiento de las hernias, y evidenciar qué coste dificultaría la sostenibilidad sanitaria ${ }^{2-8}$.

El tratamiento de las hernias ha variado desde hace 2 siglos. Además, al desarrollo de nuevas técnicas, tanto quirúrgicas (abiertas, laparoscopia) como de hospitalización (con ingreso o ambulatoria), ha colaborado el progreso de la anestesia (general, raquídea y local $)^{3,9-14}$.

\section{Objetivo}

El presente estudio se ha diseñado para conocer el coste económico del tratamiento quirúrgico de las distintas hernias de pared abdominal mediante la utilización de diferentes técnicas quirúrgicas abiertas, tipos de anestesia y estadía hospitalaria, así como los factores asociados a un aumento de dichos costes (relación coste-resultado), y de costeefectividad (valorado mediante las complicaciones $\mathrm{y}$ reintervenciones [por/sin recidiva]).

\section{Materiales y Método}

Estudio descriptivo, longitudinal, observacional y retrospectivo.

Muestra de 370 hernias de pared abdominal intervenidas, del 1 de octubre de 2015 al 30 de septiembre de 2016, por el SCG en el Hospital Universitario "Virgen de la Luz", Cuenca, hospital provincial de nivel II de Castilla la Mancha, España, con 300 camas funcionantes. Tras alta hospitalaria, revisión en consulta hasta alta definitiva.

No se incluye otro tipo de hernias (pericolostómicas), ni laparoscópicas, ni las urgentes ${ }^{1,3,12,15}$. Se excluyen hernias dobles. En anestesia, se admiten según el riesgo, desde ASA I a IV, y según su aplicación, local, raquídea y general (con independencia en su aplicación de la clasificación ASA). Y comprende intervenidos con ingreso/hospitalización $(\mathrm{CH})$ y sin ingreso/cirugía mayor ambulatoria (CMA).

La técnica quirúrgica utilizada ha sido la hernioplastía abierta libre de tensión (técnica de Lichtenstein o Rutkow-Robbins en hernias inguinofemorales y hernioplastía subaponeurótica en el resto) y la corrección del defecto herniario ha variado, según el cirujano, tipo y tamaño de la hernia.

Los pacientes fueron clasificados en 6 grupos según la localización de la hernia (inguinal, crural, umbilical, epigástrica, Spiegel, eventración -simple, con uno y con 2 o más drenajes-); subclasificados en tres grupos según el tipo de anestesia: general, raquídea y local con/sin sedación; separados en $\mathrm{CH}$ y CMA. Las complicaciones se dividieron en perioperatorias (intra y postoperatorio inmediato, $<24 \mathrm{~h}$ ), tempranas o precoces ( $<30$ días) y tardías (1-12 meses).

Datos para el estudio han sido: edad, sexo, antecedentes personales, fecha y facultativo de inclusión en LEQ (lista de espera quirúrgica), tipo de hernia, clasificación de la gravedad anestésica (ASA), preoperatorio, fecha y duración de la intervención, cirujano, tipo de anestesia aplicada, cierre y materiales utilizados, anatomía patológica, con ingreso o mediante CMA, demora en la intervención, distancia al hospital, evolución, complicaciones inmediatas y tardías, días de estancia, fecha de alta hospitalaria y definitiva, recidiva, estudios postquirúrgicos y reintervención.

El estudio cumple la Ley Orgánica 15/1999 española (protección de datos de carácter personal).

Información clínica codificada utilizando la CIE9MC.

Análisis estadístico mostrado como: Variable dependiente: coste de intervención (coste de anestesia, tiempo de quirófano, coste del material) y coste del proceso (coste de intervención, coste de CMA o días de ingreso hospitalario). Los costes utilizados en el estudio han sido reales, costes en compras-suministros y el calculado por contabilidad analítica -costes directos e indirectos- (años 2012-2015) ${ }^{16}$. Variables independientes (datos obtenidos para el estudio). Variables cualitativas: distribución de frecuencias. 
Variables cuantitativas: media y desviación estándar (DE), o bien con su mediana y su rango intercuartílico (RIC). Comparaciones de proporciones: prueba $\chi^{2}$ de Pearson. Comparaciones de medias: prueba T de Student. Análisis de normalidad: prueba de Kolmogorov-Smirnov. Análisis univariado de variables cualitativas: Test de la Mediana para muestras no paramétricas. Análisis univariado de variables cuantitativas: regresión lineal simple. Modelo de regresión lineal múltiple: análisis multivariante de costes de procedimiento de las variables que en el análisis univariado mostraron significación estadística $(\mathrm{p}<0,05)$. Dummys: variables cualitativas de más de 2 categorías. Riesgos Relativos: intervalos de confianza al 95\%. En todos los contrastes de hipótesis se asume un error a de $5 \%(\mathrm{p}<0,05)$.

Datos recogidos en hoja de cálculo Excel y el análisis estadístico se ha realizado con el paquete estadístico SPSS $21.0^{\circ}$ (SPSS Inc., Chicago, IL, EE.UU.).

\section{Resultados}

De 370 intervenciones se excluyeron 10 (hernias dobles); $\mathrm{N}$ final de 360 intervenciones, que corresponden a 359 pacientes (un paciente fue intervenido 2 veces en el periodo de estudio). La distribución por sexos fue 79,4\% hombres y $20,6 \%$ mujeres. Las edades oscilaron entre 11 años (intervención de Lichtenstein) y 91, con una media de 59,9 años (DE: 15,7). No hubo fallecidos en esta serie.

Las características sociodemográficas de la muestra, en función del riesgo anestésico del paciente (clasificación ASA) y el tipo de anestesia admi- nistrada (local y sedación $55,8 \%$, raquídea $32,2 \%$, general $11,9 \%$ ) se presentan en la Tabla 1 .

La Tabla 2 presenta variables sociodemográficas (edad, sexo) y el tiempo de duración de cirugía de las hernias intervenidas, según localización. Los tiempos quirúrgicos medios: eventración 77,3 minutos (DE: 34,6); eventración sin drenaje 40 (DE: $11,4)$, con un drenaje 79,2 (DE: 27,3), con 2 o más drenajes 121,2 minutos (DE: 45).

Intervenidas 215 hernias inguinales (202 unilaterales $(59,4 \%$ derechas $-58,3 \%$ indirectas-; $40,6 \%$ izquierdas $-76,8 \%$ indirectas-), 13 bilaterales) y 7 crurales; 28 (7,8\%) recidivadas $(67,9 \%$ inguinales).

Intervenidos por CMA 196 pacientes $(54,4 \%)$ y $\mathrm{CH} 164(45,6 \%)$, estancia hospitalaria entre 2 y 35 días (mediana de 3 días [RIC P25-P75: 2-6]) (Figura 1).

Por localización de las hernias y tipo de atención recibida, ambulatoria (CMA) o con ingreso $(\mathrm{CH})$, las hernias inguinales se atienden por CMA $(53,9 \%)$ o $\mathrm{CH}(46,1 \%)$; las crurales, umbilicales y epigátricas/supraumbilicales mayoritariamente por CMA $(85,7 \%, 72 \%$ y $65,4 \%$ respectivamente); las eventraciones y Spiegel mayoritariamente $\mathrm{CH}(88,6 \%$ y $80 \%$ respectivamente).

El $80,1 \%$ de los pacientes no presentaron complicación; el 2,3\% presentó complicaciones perioperatorias (intraoperatorias o postoperatorias), el 4,8\% complicaciones tempranas (primer mes postintervención) y el $12,8 \%$ complicaciones tardías (1-12 meses postintervención). Ningún paciente presentó complicaciones perioperatorias, tempranas y tardías simultáneamente. Hubo 3 reintervenciones por recidiva (1 hernia inguinal, 1 crural y 1 eventración con obstrucción intestinal, 5 meses después) (Figura 2).

Tabla 1. Características sociodemográficas de la muestra según categoría ASA

\begin{tabular}{|c|c|c|c|c|c|c|c|}
\hline ASA & $\mathbf{n}$ & $(\%)$ & Edad media (DE) & Sexo & n $\quad(\%)$ & Edad media (DE) & p* \\
\hline \multirow[t]{2}{*}{ ASA I } & 49 & $(13,6 \%)$ & $41,1 \quad(12,6)$ & Hombre & $35(71,4 \%)$ & $43,14(11,4)$ & \multirow[b]{2}{*}{$>0,05$} \\
\hline & & & & Mujer & $14(28,6 \%)$ & $35,93(14,3)$ & \\
\hline \multirow[t]{2}{*}{ ASA II } & 187 & $(51,9 \%)$ & $58,3(13,8)$ & Hombre & $156(83,4 \%)$ & $58,96(13,9)$ & \multirow{2}{*}{$>0,05$} \\
\hline & & & & Mujer & $31(16,6 \%)$ & $55,29(13,5)$ & \\
\hline \multirow[t]{2}{*}{ ASA III } & 101 & $(28,1 \%)$ & $69,8(11,9)$ & Hombre & $78(77,2 \%)$ & $69,45(12,2)$ & \multirow{2}{*}{$>0,05$} \\
\hline & & & & Mujer & $23(22,8 \%)$ & $71,00(11,2)$ & \\
\hline \multirow[t]{2}{*}{ ASA IV } & 23 & $(6,4 \%)$ & $70,9 \quad(9,5)$ & Hombre & $17(73,9 \%)$ & $71,71 \quad(8,6)$ & \multirow{2}{*}{$>0,05$} \\
\hline & & & & Mujer & $6(26,1 \%)$ & $68,83(12,4)$ & \\
\hline \multirow[t]{2}{*}{ Global } & \multirow[t]{2}{*}{360} & $(100 \%)$ & $59,9(15,7)$ & Hombre & $285(79,2 \%)$ & $60,64(15,2)$ & \multirow{2}{*}{$>0,05$} \\
\hline & & & & Mujer & $74(20,8 \%)$ & $57,61 \quad(17,8)$ & \\
\hline
\end{tabular}

\footnotetext{
*P: Prueba T de Student.
} 


\section{ARTíCULO ORIGINAL}

Tabla 2. Edad, sexo y duración de la intervención según tipo de hernia

\begin{tabular}{|c|c|c|c|c|c|c|}
\hline Tipo hernia & n & $(\%)$ & Años edad media (DE) & Minutos duración media (DE) & Sexo & n $\quad(\%)$ \\
\hline \multirow[t]{2}{*}{ Inguinal } & 215 & $(59,7 \%)$ & $62,4(15)$ & 55,5 (19) & Hombre & $192(89,3 \%)$ \\
\hline & & & & & Mujer & $23(10,7 \%)$ \\
\hline Crural & & & & & Mujer & $3(42,9 \%)$ \\
\hline Umbilical & 72 & $(20 \%)$ & $53,6(14)$ & 44,9 (14) & Hombre & $58(80,6 \%)$ \\
\hline \multirow[t]{2}{*}{ Epigástrica/supraumbilical } & 26 & $(7,2 \%)$ & $52(16)$ & $51,3(17)$ & Hombre & $13(50 \%)$ \\
\hline & & & & & Mujer & $13(50 \%)$ \\
\hline \multirow[t]{2}{*}{ Eventración } & 35 & $(9,7 \%)$ & $62,9(13)$ & $77,3(35)$ & Hombre & $16(45,7 \%)$ \\
\hline & & & & & Mujer & $19(54,3 \%)$ \\
\hline Global & & & & & Mujer & $74(20,6 \%)$ \\
\hline
\end{tabular}

Figura 1. Mediana estancia ingreso.

\begin{tabular}{|c|c|c|c|c|c|c|}
\hline & $2-4$ & $2-2$ & $2-7$ & $2-6$ & $5-11$ & 4-4 \\
\hline 12 & & & & & & \\
\hline 10 & & & & & & \\
\hline 8 & & & & & 6,5 & \\
\hline 6 & & & 5 & & & 4 \\
\hline 4 & 3 & 2 & & 3 & & \\
\hline 2 & & & & & & \\
\hline 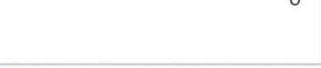 & Inguinal & Crural & Umbilical & $\begin{array}{c}\text { Epigástrica- } \\
\text { supraumbilical }\end{array}$ & Eventración & Spiegel \\
\hline Mediana estancia ingreso P50 & 3 & 2 & 5 & 3 & 6,5 & 4 \\
\hline Mediana estancia ingreso RIC & 2-4 & $2-2$ & $2-7$ & $2-6$ & $5-11$ & 4-4 \\
\hline
\end{tabular}

Las perioperatorias ingresaron y 4 tempranas (o se alargó su estancia).

Alta de cirugía 343 pacientes $(95,3 \%)$, tiempos entre 3 y 26 semanas (media 6,6 semanas).

A partir del desglose de costes (Tabla 3) se ha calculado el coste de la intervención quirúrgica y el coste del procedimiento.

El coste del material de hernioplastía (inguinal, umbilical, epigástrica y Spiegel), cada paciente, entre 109,9€ y 200,2 €. Las eventraciones sin drenaje aspirativo entre $109,9 €$ y $298,3 €$. Con un drenaje, desde $145,9 €$ hasta $334,4 €$. Si utilizan 2 drenajes, entre $182 €$ y $370,4 €(442,2 €$ por 4 Redon) (Tabla
4).

Coste hora quirófano $307 €(175,6 €$ gastos personal y $131,4 €$ gastos fijos).

Tiempos quirúrgicos medios 55,2 minutos (DE: 22).

Medianas de coste del tiempo de quirófano: hernia inguinal simple 307,2 € (RIC: $230,4-307,2$ ); crural 307,2 € (RIC: 230,4-307,2); umbilical 230,4 $€$ (RIC: 160-307,2); epigástrica 230,4 € (RIC: 204,8-307,2); Spiegel 307,2 € (RIC: 268,8-371,2); eventración 332,8 $€$ (RIC: 256-537,6). Mediana total $338,8 €$.

Coste medio por día Unidad de CMA 807,3 €: 


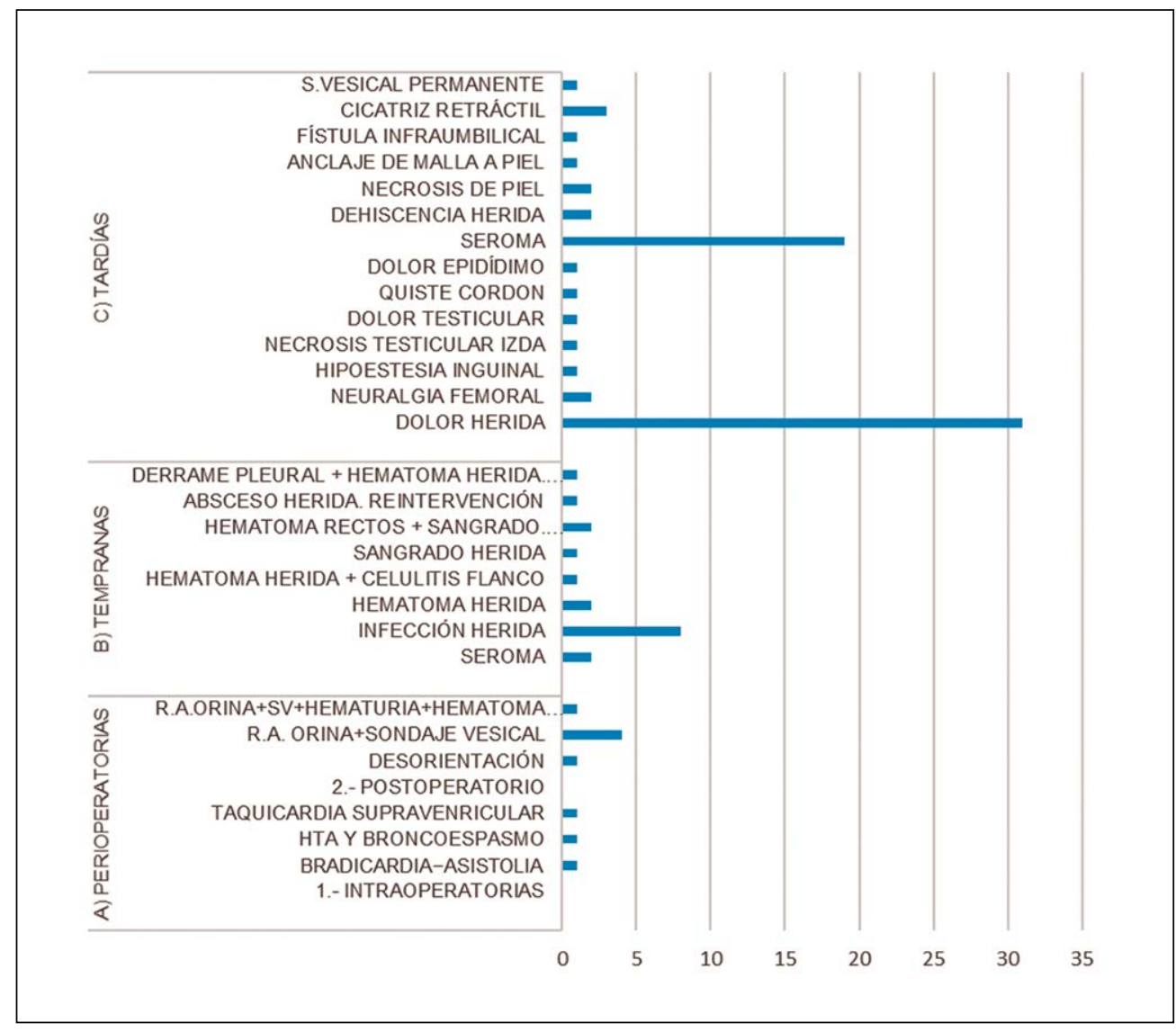

Figura 2. Complicaciones.

hernias inguino-femorales (GRD 162 y peso 0,825 ) $728,9 €$; resto de hernias (GRD 160 y peso 1,002 ) $885,7 €$

Coste medio por día de ingreso en Unidad de Hospitalización 1.056,1 €: hernias inguino-femorales (GRD 162 y peso 0,825$) 1.297,2 €$; resto de hernias (GRD 160 y peso 1,002) 703,1 $€$; hernia menores de 14 años (GRD 163) 2.156,27 $€$.

La mediana de coste por proceso con CMA es de $1.214 €$ у CH $3.690 €$.

En Tabla 4, resumen de los costes. Mediana de coste de intervención $422,7 €$ y mediana de coste de procedimiento $1.281,2 €$.

El coste mediano por intervención de hernia recidivada (19 inguinales) fue de 459,4 $€$ (RIC: $404,5-$ $575,4)$ y el coste mediano del procedimiento fue de $2.621 €$ (RIC: 1.286-7.244).

La Tabla 5 muestra los resultados del análisis univariado de las variables cualitativas y qué aumento de coste supone cada una de sus respectivas categorías. Con respecto al análisis univariado de las variables cuantitativas (edad, duración de la
Tabla 3. Desglose de costes

\begin{tabular}{|llc|}
\hline Elemento & & Euros \\
Coste & Local y sedación & 2,66 \\
& Raquídea & 24,88 \\
Coste tiempo & General & 127,20 \\
quirófano & 1 minuto & 306,97 \\
Coste medio & Malla hernia inguinal unilateral (directa o indirecta) & 5,12 \\
material & Malla hernia inguinal mixta & 112,83 \\
& Malla hernia umbilical & 138,57 \\
& Malla hernia crural & 143,16 \\
& Malla hernia epigástrica & 122,47 \\
& Malla spiegel & 199,22 \\
& Eventración simple (sin drenaje) & 279,39 \\
& Eventración drenaje 1 & 147,71 \\
& Eventración drenaje 2 & 275,49 \\
Coste CMA/ & Día de ingreso & 388,44 \\
ingreso & CMA & 1056,03 \\
& & 807,30 \\
\hline
\end{tabular}


Tabla 4. Coste de intervención y del procedimiento por cada tipo de hernia

\begin{tabular}{|c|c|c|c|c|c|c|}
\hline \multicolumn{3}{|c|}{ Localización hernia } & \multicolumn{2}{|c|}{ Coste de intervención } & \multicolumn{2}{|r|}{ Coste de procedimiento } \\
\hline & & & $\mathbf{n}$ & $\begin{array}{l}\text { Precio (euros) } \\
\text { P50 (P25-P75) }\end{array}$ & $\mathbf{n}$ & $\begin{array}{l}\text { Precio (euros) } \\
\text { P50 (P25-P75) }\end{array}$ \\
\hline \multirow[t]{3}{*}{ Inguinal } & Unilateral & $\begin{array}{l}\text { Directa/indirecta } \\
\text { Mixta }\end{array}$ & $\begin{array}{r}178 \\
24\end{array}$ & $\begin{array}{l}422,7(342,5-444,9) \\
459,5(371,6-470,7)\end{array}$ & $\begin{array}{r}172 \\
24\end{array}$ & $\begin{array}{l}1.230 \quad(1178,8-3.478,6) \\
2.582,7(1.255,7-3.625,9)\end{array}$ \\
\hline & Bilateral & $\begin{array}{l}\text { Directa/indirecta }+ \text { directa/indirecta } \\
\text { Mixta }+ \text { mixta } \\
\text { Mixta }+ \text { directa/indirecta }\end{array}$ & $\begin{array}{l}9 \\
1 \\
2\end{array}$ & $\begin{array}{l}634,5(532,1-777,1) \\
609,2 \\
865,1(788,3-941,8)\end{array}$ & $\begin{array}{l}9 \\
1 \\
2\end{array}$ & $\begin{array}{l}2.669,8(1.441,8-4.484,2) \\
3.777,3 \\
3.380,7(1.595,6-5.165,9)\end{array}$ \\
\hline & Total & & 214 & $422,7(345,9-448,3)$ & 208 & $1.341,8(1.230-3.510,7)$ \\
\hline \multicolumn{3}{|l|}{ Crural } & 7 & $432,3(329,9-432,3)$ & 7 & $1.239,6(1.137,2-1.239,6)$ \\
\hline \multicolumn{3}{|c|}{ Umbilical } & 72 & $376,2(3251-453,1)$ & 70 & $1.183,6(1.132,3-2.497,7)$ \\
\hline \multicolumn{3}{|c|}{ Epigástrica/supraumbilical } & 26 & $468,9(425,9-530,1)$ & 24 & $1.301,8(1.220,4-2.569,1)$ \\
\hline \multicolumn{3}{|c|}{ Eventración } & 35 & $709,9(585,4-863,6)$ & 34 & $7.046,1(4.382,2-1.0639,7)$ \\
\hline \multicolumn{3}{|l|}{ Spiegel } & 5 & $611,5(573,1-675,5)$ & 4 & $4.822,8(2.228,2-6.515,6)$ \\
\hline \multicolumn{3}{|l|}{ Total } & 359 & $422,7(350,6-496,1)$ & 347 & $1.281,2(1.183,5-3.613)$ \\
\hline
\end{tabular}

Tabla 5. Análisis univariado para costos de intervención y costos de proceso según variables cualitativas independientes

\begin{tabular}{|c|c|c|c|c|c|c|c|c|c|}
\hline \multirow{2}{*}{\multicolumn{2}{|c|}{ Variable independiente }} & \multicolumn{4}{|c|}{ Coste de intervención } & \multicolumn{4}{|c|}{ Coste procedimiento } \\
\hline & & \multirow{2}{*}{$\begin{array}{c}\mathbf{n} \\
285\end{array}$} & \multirow{2}{*}{$\begin{array}{c}\text { Mediana } € \\
422,7\end{array}$} & \multirow{2}{*}{$\begin{array}{c}\text { P50 } \\
(350,6-470,6)\end{array}$} & \multirow{2}{*}{$\begin{array}{l}\mathbf{p}^{*} \\
0,167\end{array}$} & \multirow{2}{*}{$\begin{array}{c}\mathbf{n} \\
276\end{array}$} & \multirow{2}{*}{$\begin{array}{c}\text { Mediana } € \\
1.262,6\end{array}$} & \multirow{2}{*}{$\begin{array}{c}\text { P50 } \\
(1.183,5-3.583,1)\end{array}$} & \multirow{2}{*}{$\begin{array}{l}\mathbf{p}^{*} \\
0,409\end{array}$} \\
\hline Sexo & Hombre & & & & & & & & \\
\hline \multirow[t]{3}{*}{ ASA } & ASA I & 49 & 422,7 & $(345,9-446,6)$ & 0,035 & 47 & 1.230 & $(1.153,2-1.260,3)$ & $<0,001$ \\
\hline & ASA II & 186 & 422,8 & $(354,9-484,7)$ & & 183 & $1.234,7$ & $(1.162,8-2.633,9)$ & \\
\hline & ASA IV & 23 & 453,1 & $(342,5-607,6)$ & & 23 & 5.166 & $(3.459,4-7999,8)$ & \\
\hline \multirow[t]{3}{*}{ Anestesia } & Local y sedación & 201 & 422,8 & $(345,9-432,3)$ & $<0,001$ & 197 & 1.230 & $(1.153,2-1.255,6)$ & $<0,001$ \\
\hline & Raquídea & 115 & 444,9 & $(368,1-521,8)$ & & 109 & 3.613 & $(2.480,2-4.994,2)$ & \\
\hline & General & 43 & 577,6 & $(500,8-812,3)$ & & 41 & $5.036,4$ & $(2.689,6-8.710,1)$ & \\
\hline $\begin{array}{l}\text { Tipo de } \\
\text { ingreso }\end{array}$ & CMA & 196 & 406,7 & $(345,9-432,3)$ & $<0,001$ & 196 & 1.214 & $(1.153,2-1.239,6)$ & $<0,001$ \\
\hline \multirow{5}{*}{$\begin{array}{l}\text { Localización } \\
\text { hernia }\end{array}$} & Crural & 7 & 432,3 & $(329,9-432,3)$ & & 7 & $1.239,6$ & $(1.137,2-1.239,6)$ & \\
\hline & Umbilical & 72 & 376,2 & $(325,1-453,1)$ & & 70 & $1.183,5$ & $(1.132,3-2.497,7)$ & \\
\hline & Epigástrica/supraumbilical & 26 & 468,9 & $(425,9-531,2)$ & & 24 & $1.301,8$ & $(1.220,4-2.569,1)$ & \\
\hline & Eventración & 35 & 709,9 & $(585,3-863,6)$ & & 34 & $7.046,1$ & $(4.382,2-1.0639,7)$ & \\
\hline & Spiegel & 5 & 611,5 & $(573,1-675,5)$ & & 4 & $4.822,8$ & $(2.228,2-6.515,6)$ & \\
\hline \multirow{4}{*}{$\begin{array}{l}\text { Complica- } \\
\text { ciones }\end{array}$} & Ninguna & 282 & 422,7 & $(345,9-470,6)$ & 0,058 & 278 & $1.260,3$ & $(2.309,8-6.828,1)$ & 0,001 \\
\hline & Perioperatoria & 7 & 475,2 & $(419,3-633,2)$ & & 6 & $5.173,8$ & $(2.802,6-1.6297,5)$ & \\
\hline & Temprana & 17 & 526,4 & $(375,1-686,3)$ & & 16 & $5.900,7$ & $(2.802,6-1.6297,6)$ & \\
\hline & Tardía & 45 & 432,3 & $(357-567,1)$ & & 44 & $1.288,4$ & $(1.195,1-3.659,2)$ & \\
\hline
\end{tabular}

*Test no paramétrico de la mediana. 
Tabla 6. Riesgo relativo

\begin{tabular}{|llcrrr|}
\hline Variable independiente & & RR & \multicolumn{2}{c}{ IC 95\% } & \multicolumn{1}{c|}{ Sig. } \\
ASA & & & Inf & Sup & 0,769 \\
& ASA II & 158,6 & $-904,6$ & $1.221,8$ & 0,338 \\
& ASA III & 608,9 & $-638,4$ & $1.856,2$ & 0,047 \\
Anestesia & ASA IV & $1.771,6$ & 21,9 & $3.521,2$ & 0,663 \\
& Raquídea & 247,1 & $-868,8$ & $1.363,1$ & 0,001 \\
Tipo de ingreso & General & 2.284 & 955,9 & $3.612,1$ & 0,000 \\
Duración de la intervención & Ingreso & $2.937,3$ & $1.836,8$ & $4.037,8$ & 0,000 \\
\hline
\end{tabular}

Tabla 7. Costes procedimiento según localización de la hernia y complicaciones

\begin{tabular}{|llll|}
\hline Localización hernia & & Complicaciones & Sí \\
\hline Inguinal & $\mathrm{n}(\%)$ & $177(84,29 \%)$ & $33(15,71 \%)$ \\
& Coste P50 (P25-P75) & $1297.46(1227.46-3513.17)$ & $1405.81(1229.99-3510.60)$ \\
Crural & $\mathrm{n}(\%)$ & $4(57,14 \%)$ & $3(42,86 \%)$ \\
& Coste P50 (P25-P75) & $1201.23(1105.23-1239.63)$ & $1239.63(1137.23-2566.61)$ \\
\hline Umbilical & $\mathrm{n}(\%)$ & $57(81,43 \%)$ & $13(18,57 \%)$ \\
& Coste P50 (P25-P75) & $1183.52(1113.12-1260.32)$ & $1260.32(1157.92-7365.00)$ \\
Epigátrica/supraumbilical & $\mathrm{n}(\%)$ & $20(76,92 \%)$ & $6(23,08 \%)$ \\
& Coste P50 (P25-P75) & $1239.58(1213.98-2566.56)$ & $1469.98(1277.98-9006.79)$ \\
Eventración & $\mathrm{n}(\%)$ & $19(55,88 \%)$ & $15(44,12 \%)$ \\
& Coste P50 (P25-P75) & $5865.50(2770.75-7999.78)$ & $8350.58(7046.07-13447.28)$ \\
Spiegel & $\mathrm{n}(\%)$ & $5(100 \%)$ & $0(0 \%)$ \\
\hline
\end{tabular}

intervención y duración de la estancia), todas ellas mostraron asociación estadísticamente significativa con los costes de procedimiento y sólo la duración de la intervención y la duración de la estancia mostraron asociación estadísticamente significativa con los costes de intervención.

La Tabla 6 muestra los Riesgos Relativos (RR), con sus intervalos de confianza al 95\% (IC 95\%), de aquellas variables que mostraron asociación estadísticamente significativa con el coste del procedimiento en el modelo final de regresión lineal, que obtuvo una R cuadrada ajustada de 0,44.

Los costes derivados de las complicaciones producidas por la anestesia general (incremento por tres) son muy superiores a los producidos por la local y la espinal. La Tabla 7 muestra los costes de las complicaciones producidas según el tipo de hernia.

Coste total de las consultas (anestesia, cirugía, postquirúrgica) y curas de enfermería ha ascendido a $131.497,8 €$ (media/paciente, $355,4 €$ ). Coste total de las complicaciones postquirúrgicas tras alta hospitalaria $28.547,5 €$.

\section{Discusión}

Las políticas sanitarias, como objetivo primordial, están orientadas a proveer servicios médicos a los ciudadanos en tiempo, coste y calidad lo mejor posible. Pero para cumplir este objetivo y, además, garantizar la sostenibilidad del sistema sanitario 
español actual (universal, público, "gratuito” y de calidad) hay que hacer una utilización eficiente de los recursos disponibles (hecho éste que se debe aprender, y no olvidar, tras la grave crisis económico-social de estos últimos años). Y el hospital público debe cumplir todos estos requisitos ${ }^{2,3,5}$. Por ello, las acciones tomadas encaminadas a estos fines, deben basarse en datos y números, que ratifican de manera objetiva los hechos acaecidos, siendo fundamental la contabilidad analítica (favorece una eficiente gestión clínica y financiera, y permite conocer las necesidades reales y el volumen de actividad asistencial), y la realización de estudios de rentabilidad $3,5,17,18$.

En los SCG los GRD 160 (procedimientos sobre hernia no inguinal) y 162 (procedimientos sobre hernia inguinal) son intervenciones muy frecuentes.

En nuestro estudio, de 360 hernioplastías y seguimiento máximo 12 meses, el 79,4\% varones, 60,1 años de media, 51,9\% ASA II, 90,5\% profilaxis antibiótica, 55,8\% anestesia local, 59,7\% hernias inguinales, $36,9 \%$ hernias inguinales unilaterales indirectas, 54,4\% por CMA, 4,8 días de estancia media (mediana: 3 ) en ingresados, $76 \mathrm{~km}$ de distancia media de pacientes procedentes de fuera de la capital, altas $95,4 \%$ con tiempo medio 6,6 semanas. Complicaciones perioperatorias 2,3\%, tempranas $4,8 \%$ (con 5 reintervenciones) y tardías 12,8\% (3 reintervenciones por recidiva). Cifras que difieren de estudios previos $^{6,12,17-19,20}$. No hubo fallecidos. Aparecieron recidivas entre 3 y 9 meses, con porcentajes similares, como en otros estudios ${ }^{6,18-20}$.

Del total de intervenciones, 59,7\% fueron hernias inguinales, 94\% unilaterales $(65,8 \%$ indirectas; $22,3 \%$ directas; $11,8 \%$ mixtas, $89,3 \%$ varones, 62,4 años de edad media; 44,6\% ASA II; 50,7\% anestesia local) y el 1,9\% crurales-femorales, siendo cifras parecidas a otros estudios ${ }^{21}$.

La demora para la intervención de hernia (media 93,2 días; DE: 43,4) se incrementó respecto a años anteriores.

Los pacientes ASA II-III fueron los más frecuentes, interviniéndose mayor número en CMA y sólo 4 reingresos tras el alta. Las complicaciones, según tipo de anestesia, han sido inversamente proporcionales al número total aplicado, más numerosas, porcentualmente, con anestesia general y menos con local ${ }^{5,14,22-24}$.

El coste/día paciente CMA ha sido $807,3 €$ y con ingreso $1.056,1 €$. Comparados con 2001, incremento de $441,5 €$ y $478,9 €$ respectivamente ${ }^{5,9,16}$. Diferencia de costes marcada por la estancia hospitalaria; no hay en CMA, lo que permite reducir costes por proceso (3.689,8 € con hospitalización) $)^{2,10,12,24}$.
Las hernioplastías que han mostrado costes más elevados han sido, por orden de mayor a menor según su localización: eventraciones, Spiegel, epigástrica/supraumbilical, crural, inguinal y umbilical ${ }^{3,15}$, si bien las diferencias entre ellas no son estadísticamente significativas. Además, las hernias inguinales, las más frecuentes de todas, presentan costes muy distintos según se trate de hernias unilaterales (costes de procedimiento: $1.230 €$ hernia unilateral directa/indirecta y $2.582,7 €$ hernia unilateral mixta) o bilaterales (entre 2.669,8 $€$ y $3.777,3 €$ de costes de procedimiento).

En nuestro estudio, la anestesia local fue más aplicada y los costes medios por procedimiento menores que en estudios previos ${ }^{2,14}$.

La intervención de hernia inguinal unilateral simple, crural y umbilical con malla, anestesia local y mediante CMA es más coste efectiva ${ }^{2,7,8,11,13,25}$. No hay comparación con hernioplastías laparoscópicas, al no ser realizadas en el hospital ${ }^{3,4,12,15}$, ni con estudios en hernias crurales $2,11,13,25$.

Los resultados de este estudio muestran que la anestesia local fue la más utilizada, la mayoría pacientes ASA II-III y presencia de complicaciones precoces, con independencia del tipo de anestesia aplicada, que difiere de otros estudios ${ }^{2,14,25}$. Ocurriendo complicaciones tempranas y tardías, y reintervenciones, en pacientes con anestesia regional y general $^{2,18}$. La anestesia local permite realizar más intervenciones mediante CMA, tiene menos costes y complicaciones $2,5,9,11,13,14,19,25$.

Los costes de los procedimientos en relación con los costes por intervención se ven más incrementados en pacientes ASA III y IV (por 8 y por 11) respecto a los ASA I y II (por 3); aplicando anestesia raquídea y general (por 9) respecto a la local (por 3); en las eventraciones y Spiegel (por 10 y por 8 ) respecto al resto de hernias (por 3 ); y por complicaciones perioperatorias e inmediatas (por 11) respecto a ninguna complicación o tardías (por 3 ), como en otros estudios ${ }^{2,5,13}$ y difiere de estudios previos $^{12,14,19}$.

Los factores que en nuestro estudio han mostrado tener un efecto estadísticamente significativo en el aumento de los costes por procedimiento han sido: riesgo anestésico ASA IV (incremento de 1.771,6 $€$ respecto a ASA I), recibir anestesia general (incremento de $2.284 €$ respecto a anestesia local), necesitar hospitalización (incremento de 2.937,3€ respecto a CMA) y mayor duración de la intervención (incremento de $71,4 €$ por cada minuto más de cirugía). Otros factores como el sexo del paciente, el tipo de hernia o las complicaciones no han mostrado efecto estadísticamente significativo. 


\section{Conclusión}

Las intervenciones de hernia inguinal unilateral simple, crural y umbilical, con utilización de una técnica libre de tensión y material protésico sin drenaje, en régimen de CMA, con anestesia local (con/sin sedación) y sin complicaciones resultan las más coste-efectivas (mejor relación coste-beneficio y coste-efectividad). Las intervenciones en pacientes ASA IV, con anestesia general, con mayor duración quirúrgica e ingreso hospitalario son las más costosas (menos coste-efectivas).

\section{Responsabilidades éticas}

Protección de personas y animales. Los autores declaran que para esta investigación no se han realizado experimentos en seres humanos ni en animales.

Confidencialidad de los datos. Los autores declaran que en este artículo no aparecen datos de pacientes.

Conflictos de interés: no hay.

\section{Bibliografía}

1. Randhawa SK, Centrulo LN, Fathalizadeh A, Leung PS, Bilsland D, Joshi AR. Laparoscopic total extraperitoneal (TEP) repair inguinal hernias using manual dissection instead of a balloon dissectorpotential cost savings. Surg Endosc Interv Tech. 2016;30:S406.

2. De Miguel Ibáñez R, Nahban al Saied SA, Alonso Vallejo J, Rodríguez Canales JM, Blanco Prieto C, Escribano Sotos F. Cost-effectiveness of primary abdominal wall hernia repair in a 364-bed provincial hospital of Spain. Hernia 2011;15:377-85.

3. Fernández Lobato R, Ruiz de Adana JC, Angulo Morales F, García Septiem J, Marín Lucas FJ, Limones Esteban M. Estudio de coste-beneficio comparando la reparación de la hernia ventral abierta y laparoscópica. Cir Esp. 2014;92:553-60.

4. Vale L, Grant A, McCormack K. Costeffectiveness of alternative methods of surgical repair of inguinal hernia. Int $\mathrm{J}$ Technol Assess Helth Care 2007;20:192200.

5. Vila Blanco JM. Gestión de costes mediante la implantación de una unidad de cirugía mayor ambulatoria. Cir May Amb. 2002;7:66-72.

6. Löfgren J, Nordin P, Ibingira C, Matovu A, Galiwango E, Wladis AA. Randomized trial of low-cost mesh in groin hernia repair. N Engl J Med. 2016;374:146-53.

7. Plisko R, Metz L, Dzieviatka M. Costeffectiveness of alternative methods of surgical repair of inguinal hernia. Int $\mathrm{J}$ Technol Assess Health Care. 2004;20: 926.
8. Plisko R, Metz L, Dzieviatka M. Cost-effectiveness comparison of tension free mesh repair $v s$ tension suture methods of inguinal hernia in Slovakia. Hungary. Poland. Value Health. 2008;11: A243-A244.

9. Vila Blanco JM. Desarrollo e implantación de la CMA en España: evolución en los hospitales del INSALUD. Cir Esp. 2004;76:177-83.

10. Vila Blanco JM, Sáiz Jarabo JM. Implantación de la cirugía mayor ambulatoria en un hospital general. Cir May Amb. 2009;14:99-102.

11. Sturniolo G, Bonanno L, Lo Schiavo MG, Tonante A, Taranto F, Gagliano E, et al. Day-surgery as a factor in reducing hospital stay. Chirurgia Italia. 2007;59:4152.

12. Langeveld HR, Van't Riet M, Weidema WF, Stassen LPS, Steyerberg EW, Lange J, et al. Total extraperitoneal inguinal hernia repair compared with Lichtenstein. Ann Surg. 2010;251:819-24.

13. Nordin P, Zetterstrom H, Carlsson P. Costeffectiveness analysis of local, regional and general anaesthesia for inguinal hernia repair using data from randomized clinical trial. Br J Surg. 2007;94:500-5.

14. O’Dwyer PJ, Serpell MG, Millar K, Paterson C, Young D, Hair A, et al. Local or general anesthesia for open hernia repair: a randomizad trial. Ann Surg. 2003;237:574-9.

15. Ecker BL, Kuo L, Simmons KD, Fischer JP, Morris JB, Kelz RR. Laparoscopic versus open ventral hernia repair: One year outcomes and cost analysis using state-wide claims data. Surg Endosc and Other Interv Techn. 2015;29:S328.

16. Dirección de Gestión. Contabilidad Analítica. Hospital Universitario "Virgen de la Luz". Cuenca. 2015.

17. Rodríguez-Cuéllar E, Villeta R, Ruiz P, Alcalde J, Landa JI, Porrero JL, et al. Proyecto nacional para la gestión clínica de procesos asistenciales. Tratamiento quirúrgico de la hernia inguinal. Cir Esp. 2005;77:194-202.

18. Parrilla Paricio P, Landa García JI. Cirugía AEC $2^{\mathrm{a}}$ edición. Madrid: Ed. Panamericana. 2010. J.I. Landa García. A. Muñoz-Calero Peregrín. Complicaciones de las hernias de la región inguinocrural: 219-23.

19. Gianetta E, Cuneo S, Vitale B, Camerini G, Marini P, Stella M. Anterior tensionfree repair of recurrent inguinal hernia under local anesthesia: a 7-year experience in a teaching hospital. Ann Surg. 2000;231:132-6.

20. O'Dwyer PJ, Norrie J, Alani A, Walker A, Duffy F, Horgan P. Observation or operation for patiens with an asymptomatic inguinal hernia: a randomized clinical trial. Ann Surg. 2006;244:167-73.

21. Parrilla Paricio P, Landa García JI. Cirugía AEC $2^{\mathrm{a}}$ edición. Madrid: Ed. Panamericana. 2010. F.Carbonell Tatay. X. Feliu Palá. Hernias de la región inguinocrural: 203-18.

22. Monedero P, Errando C, Adam M. Grupo de Revisión de los Protocolos sobre Seguridad de los Pacientes en Anestesiología. La Declaración de 


\section{ARTÍCULO ORIGINAL}

Helsinki sobre seguridad de los pacientes en anestesiología. Rev Esp Anestesiol Reanim. 2013;60 (Supl 1):1-3.

23. Zaballos M, López-Álvarez S, Argente P, López A. Recomendaciones de pruebas preoperatorias en el paciente adulto para procedimientos en régimen de cirugía ambulatoria. Rev Esp Anestesiol Reanim. 2015;62:29-41.

24. Viñoles J, Argente P. Criterios de alta en cirugía ambulatoria. Cir May Amb. 2013;18:125-32.
25. De Miguel Ibáñez R, Nahban-al Saied SA, Alonso Vallejo J. Escribano Sotos F. Resultados de satisfacción y calidad de vida percibida en pacientes intervenidos de hernia primaria de pared abdominal. Cir Esp. 2015;93:658-64. 